\title{
Spatio-temporal epidemiology of emergency medical requests in a large urban area. A scan-statistic approach
}

\author{
Soheil Hashtarkhani, ${ }^{1}$ Behzad Kiani, ${ }^{1}$ Alireza Mohammadi, ${ }^{2}$ Shahab MohammadEbrahimi, ${ }^{1}$ \\ Mohammad Dehghan-Tezerjani, ${ }^{3}$ Tahereh Samimi, ${ }^{4}$ Mahmood Tara, ${ }^{1}$ Stephen A. Matthews ${ }^{5}$ \\ ${ }^{1}$ Department of Medical Informatics, School of Medicine, Mashhad University of Medical Sciences, \\ Mashhad, Iran; ${ }^{2}$ Department of Geography and Urban Planning, Faculty of Social Sciences, University of \\ Mohaghegh Ardabili, Ardabil, Iran; ${ }^{3}$ Department of Anaesthesiology and Critical Care, Shahid Sadoughi \\ University of Medical Sciences and Health Services, Yazd, Iran; ${ }^{4}$ Department of Medical Informatics, \\ School of Allied Medical Sciences, Urmia University of Medical Sciences, Urmia, Iran; ${ }^{5}$ Department of \\ Sociology and Criminology, and Department of Anthropology, The Pennsylvania State University, University \\ Park, PA, USA
}

\begin{abstract}
Pre-hospital care is provided by emergency medical services (EMS) staff, the initial health care providers at the scene of disaster. This study aimed to describe the characteristics of EMS callers and space-time distribution of emergency requests in a large urban area. Descriptive thematic maps of EMS requests were created using an empirical Bayesian smoothing approach. Spatial, temporal and spatio-temporal clustering techniques were applied to EMS data based on Kulldorff scan statistics technique. Almost 225,000 calls were registered in the EMS dispatch centre during
\end{abstract}

Correspondence: Mahmood Tara, Department of Medical Informatics, School of Medicine, Mashhad University of Medical Sciences, Mashhad, Iran. E-mail: Taram@mums.ac.ir

Key words: Geographical information systems; emergency medical service; space-time clustering; ambulance calls; Iran.

Acknowledgements: Special thanks are extended to the staff of EMS centre of Mashhad University of Medical Sciences who provided the information needed for this study.

Ethical issues: the identity of all callers was anonymous for privacy issues.

See online Appendix for additional Tables.

Received for publication: 18 August 2021.

Revision received: 22 October 2021.

Accepted for publication: 22 October 2021.

CCopyright: the Author(s), 2021

Licensee PAGEPress, Italy

Geospatial Health 2021; 16:1043

doi:10.4081/gh.2021.1043

This article is distributed under the terms of the Creative Commons Attribution Noncommercial License (CC BY-NC 4.0) which permits any noncommercial use, distribution, and reproduction in any medium, provided the original author(s) and source are credited. the study period. Approximately two-thirds of these calls were associated with an altered level of patient consciousness, and the median response time for rural and urban EMS dispatches was 12.2 and 10.1 minutes, respectively. Spatio-temporal clusters of EMS requests were mostly located in central parts of the city, particularly near the downtown area. However, high-response time clustered areas had a low overlap with these general, spatial clusters. This low convergence shows that some unknown factors, other than EMS requests, influence the high-response times. The findings of this study can help policymakers to better allocate EMS resources and implement tailored interventions to enhance EMS system in urban areas.

\section{Introduction}

Emergency medical services (EMS), also known as ambulance services, provide medical transport and/or out-of-hospital medical care to people exposed to accidents or otherwise in need of immediate medical care (Spaite et al., 2001). EMS is thus the first meeting point for people who need emergency health care, and if timely service to seriously ill patients fail, a life may be lost (Leonardsen et al., 2021). Therefore, considering the importance of timely addressing the unique needs of at-risk people, pre-hospital emergency care is one of the priorities of the global health system (Nichol et al., 2008). Given the vital role of high-quality and efficient emergency care in saving patients' lives from unpredicted life-threatening injuries or critical conditions, the EMS goal is to provide timely and well-monitored care (Aringhieri et al., 2017). To achieve this goal, having a full perspective of the EMS system including the medical characteristics and location of the callers as well as access to available resources distribution is undeniable (Wang et al., 2012).

In developing countries, high death rates and/or irreversible injuries in pre-hospital care are quite common (Mawani et al., 2016). Based on a report by Haddadi et al. (2017) more than $50 \%$ of those who have lost their lives in critical situations died at the scene and about $16 \%$ died on the way to the hospital. Patient survival rate in pre-hospital care is mainly associated with response time (Ong et al., 2009) and it has been proven that timely emergency services will improve outcomes in sensitive situations (MacKenzie et al., 2006). However, other fundamental factors, 
such as heavy traffic, poor-quality roads are the main reasons for delay in Iran, but also scattered, inadequate and under-equipped EMS stations also play a role (Haddadi et al., 2017).

In Iran, the accepted EMS response time is defined as less than 8 and $15 \mathrm{~min}$ in urban and suburban areas, respectively (Bahadori et al., 2010). However, the response time varies in different cities, e.g., in Tehran, the capital of Iran, the average response time has been reported at $12.54 \mathrm{~min}$ (Bidari et al., 2007). Another study conducted in Urmia, north-west Iran, showed a mean response time for city locations of $5 \mathrm{~min}$ and $10.6 \mathrm{~min}$ for interurban road locations (Bigdeli et al., 2010). In Yazd, central Iran, $81.2 \%$ of EMS requests were responded within the 8 min standard (Bahrami et al., 2011).

Recently, researchers and health organisations have increasingly applied spatio-temporal analysis to represent and control health-related issues (Ahmadian et al., 2020), applying geographic information systems (GIS) to explore the pattern of pandemics and health events (Nykiforuk and Flaman, 2011; Mena et al., 2018; MohammadEbrahimi et al., 2021). GIS is a highly useful tool for decision-making, and it effectively visualises space-time information in different overlays (Adin et al., 2019). Indeed, the growth of data storage and analysis technologies in the $21^{\text {st }}$ century and increasing attention to the efficiency of the emergency medical system has promoted GIS applications in EMS-related studies (Ong et al., 2009; Schuurman et al., 2009; Hashtarkhani et al., 2020; Tabari et al., 2020;). This approach is useful for visualising the distribution of EMS incidents showing that they do not follow random spatio-temporal patterns but rather have particular spacetime patterns in different geographical areas (Warden et al., 2007). Already two decades ago, Peters and Hall (1999) utilised GIS to arrange and appraise ambulance stations and their response times finding that socio-spatial characteristics were good predictors for the spatial-temporal patterns of response times. Bassil et al. (2009) focused on mapping heat-related diseases in Toronto, Canada in relation to all EMS calls made and found that the EMS medical dispatch data followed a similar pattern between the timing of the heat events and extreme heat alerts in different areas. Similarly, Ong et al. (2009) explored the geospatial patterns of EMS requests in Singapore discovering a definite spatio-temporal pattern leading them to recommend that ambulance deployment be based on realtime call monitoring. Some factors such as circadian rhythms, time of day and geographical epidemiology of the population were influential with respect to the patterns of EMS calls and ambulance demands. Along these lines, Xia et al. (2019) concluded that accessibility to EMS in the central area of the city is temporally sensitive due to large and frequent population flow based on a large volume of data from the global positioning system (GPS) and the enhanced two-step floating catchment area (E2SFCA). Thus, EMS requests follow spatio-temporal patterns, and the incidents are dynamic, especially in big cities with high mobility levels (Azimi et al., 2021). At this time, there is not enough knowledge about the spatio-temporal distribution of EMS requests in Iran's large cities. The main questions regard the pattern of EMS requests in its big cities and how the most effective EMS deployment strategy can be designed in the future.

A spatio-temporal measurement based on EMS requests distribution will enable closer attention to response times in circumstances where time delays are critical. The current study aimed to study this in Mashhad, the second-most populous city of Iran, focusing on the spatial and temporal distribution of EMS calls to identify any potential pattern(s) of ambulance enquiries. Studies along these lines have been conducted in Mashhad but only with reference to specific health conditions, such as cardiovascular dis- eases (Azimi et al., 2021) and road traffic injuries (Tabari et al., 2020). No prior work has investigated the spatio-temporal distribution of all EMS requests in the Mashhad area and the potential clustering of incidents, and we wished to demonstrate the high utility of GIS in EMS requests analysis; identify the influential factors on EMS response rate; and inform allocation decisions of EMS resources in future. We opted to carry out a retrospective cross-sectional study employed ambulance call data to discover characteristics and patterns of ambulance enquiries.

\section{Materials and methods}

\section{Study setting and population}

This ecological study was conducted in Mashhad, the capital of Razavi Khorasan Province. It is located in the northeast of the country and the latest census estimated the population at 3,785,567 (https://www.amar.org.ir/english/Population-and-HousingCensuses). Mashhad is known as a place of religious pilgrimage and is the most popular tourist destination in the country, with over 20 million visitors per annum (Kafashpor et al., 2018). Figure 1 illustrates the census tracts, population density and location of EMS stations in urban area. The downtown area includes the Holy Shrine with a high concentration of hotels, residential complexes and shopping malls.

\section{Data collection}

Two sets of data were used: i) ambulance call data from 1 June 2018 to 31 May 2019 from the database at Mashhad's EMS centre; and ii) the population's spatial distribution from the city municipality. Calls that did not lead to an ambulance mission were removed from the analysis. The EMS data also included demography information, dates and response times, the main medical condition/complaint either recorded by the nurses in the EMS dispatch centre or the ambulance staff's individual personal digital assistants (PDAs). Data for all EMS dispatches (inside and outside the city) were collected for descriptive analysis, while only requests from the city area were included for map generation and spatial analysis. Additionally, we used median and interquartile ranges to report central tendency statistics for the time mission intervals.

\section{Thematic mapping}

Smoothed cumulative incidence rates were mapped at the census tract level $(n=1301)$. The heterogeneity of the variance and spatial autocorrelation of EMS rates were adjusted for smoothing by using empirical Bayesian smoothing (EBS) (Robertson, 1999) to show the pattern of requests. This technique is suitable when the areal population size and aggregation vary and when there is spatial autocorrelation in the data (Pringle, 1996), so it was applied given that the population size varied by census area $(S D=2680)$ and the presence of spatial autocorrelation of the EMS rates (global Moran's $I=0.13$ at $\mathrm{P}<0.001$ ). The EBS smoothed incidence rates, which computes the risk as a weighted sum of the raw rate for each unit and a prior mean (Manton et al., 1989), were grouped into five categories using the natural breaks classification algorithm. For analysis of diurnal variation, we adopted the four-hour interval scheme (starting at 3:00 a.m.) used by Ong et al. (2009). A separate map was generated for each of the six periods. 


\section{Spatial and temporal clustering}

The descriptive maps show the spatial pattern of EMS requests during each time interval, but to identify the high-risk (hotspots) areas of EMS requests by time, we needed to add scan statistics introduced in health sciences by Naus (1965) with spatial and temporal extensions introduced by Kulldorff (1997). These techniques can identify spatial, temporal and space-time clustering as used by (De Carvalho et al., 2020; Punyapornwithaya et al., 2020; Silva et al., 2020). In Kulldorff's scan statistics, the first step is to determine a congruous probability model of the data and compute the likelihood ratio for each scan window. Then, the primary cluster candidates with the maximum likelihood ratio are identified and the Monte Carlo hypothesis procedure tests the statistical significance, i.e. P-value (Kulldorff, 1997). Relative risk (RR), Log likelihood ratio (LLR) and the Mont Carlo test constitute the approaches used for the interpretation of scan statistics (Kulldorff, 2018).

\section{Purely spatial cluster analysis}

Purely spatial scan statistics was used to discover spatial clusters of high EMS demand. A retrospective discrete Poisson model was used, assuming that the number of calls in each census tract is
Poisson-distributed, based on a known background population (Kulldorff, 1997). Latitude and longitude coordinates for each census centroid were calculated. The maximum spatial cluster size was set to $50 \%$ of the population at risk as recommended by Kulldorff (2018).

\section{Purely temporal cluster analysis}

Purely temporal scan statistics was performed to discover the timing of the meaningfully higher rate of EMS requests. This was done by searching for purely temporal clusters analysis without addressing their spatial variations or patterns (Kulldorff, 2018). The Poisson discrete scan statistic was set as the probability model to detect high rate time clusters of the day. The length of time aggregation was set at 4 hours (six time intervals as introduced in descriptive analysis) and the window size was set to $50 \%$.

\section{Space-time cluster analysis}

Space-time scan statistics was used to identify the geographical locations where EMS requests were significantly high at certain times of the day. A circular scanning window was selected to detect large, compact clusters. The scanning window moved across space and time simultaneously comparing the rate of EMS requests inside the scanning window to the rate of EMS requests outside of
(A) IRAN

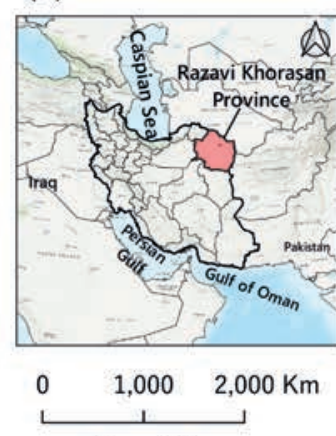

(B) Razavi-Khorasan

\section{(B)}

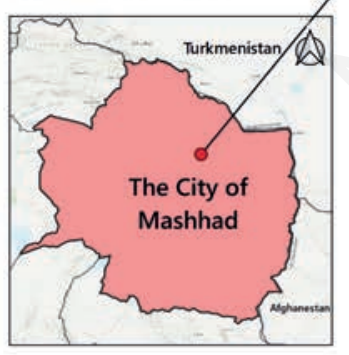

0

$300 \mathrm{Km}$

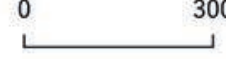

Coordinates:
$X: 59.567131 ;$ Y: 36.318779

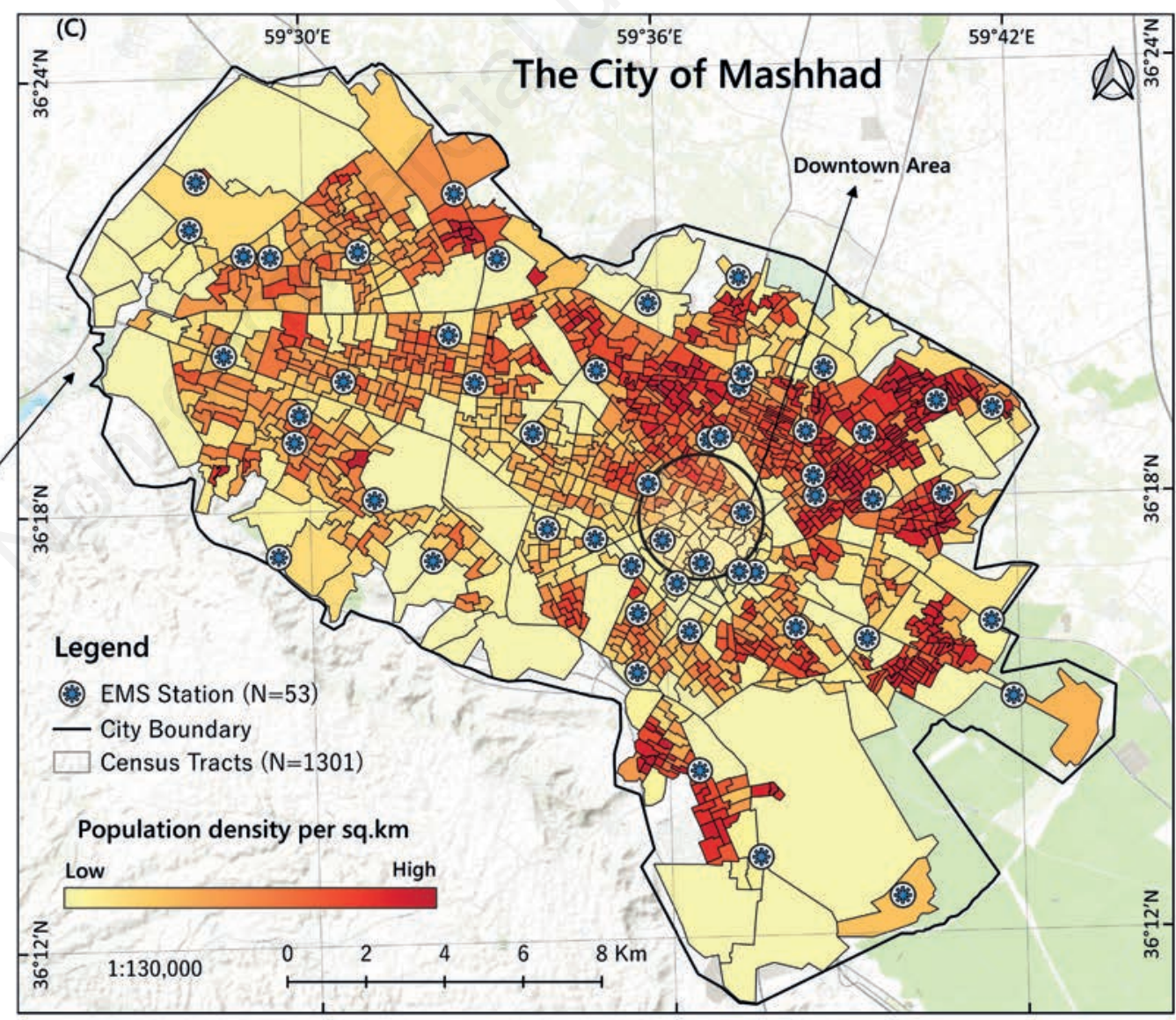

Figure 1. Map showing the population density in census tracts and the distribution of emergency medical services (EMS) ambulance stations in the city of Mashhad. 
the scanning window. If the calculated rate is significantly $(\mathrm{P} \leq 0.05)$ higher inside the window, the $\mathrm{RR}$ is estimated, and a potential high-rate cluster identified. We set the scanning window to include up to $50 \%$ of the study population and up to $50 \%$ of the study period to detect large primary and secondary clusters.

\section{Analysis of spatial variation in temporal trends}

Spatial variation in temporal trends (SVTT) is a relatively new approach, which detects clusters with relatively higher rising or falling trend over the time (Moraga and Kulldorff, 2016). This method provides an estimate of the temporal trend throughout the study area. The null hypothesis is that the trends are the same, while the alternative assumes that they are different. A cluster can have a high trend because it has a rate that increases or decreases faster than the outside area. The maximum windows size was adjusted to $50 \%$ as well.

\section{Convergence of emergency medical services requests and response time clusters}

In this step, we studied the possible relationships between the rates of EMS requests and the resulting response time of ambulance missions. To that end, the average response time of each census tract was calculated, and the hotspots of high-response time identified using the purely spatial scan statistics. Then the clusters of EMS requests were overlaid on a single map assuming that the overlapping patterns of these two would represent the probable correlation between the response time and the number of requests.

\section{Software}

GeoDA (Centre for Spatial Data Science, University of Chicago, IL, USA) was used to estimate EBS of incidence rates. QGIS, v. 3.18.2 (https://qgis.org/downloads/) and ArcGIS, v. 10.6 (ESRI, Redlands, CA, USA) was used to create the maps. We also used SaTScan v.9.7 (https://www.satscan.org/) specialised software to perform spatial, temporal and spatio-temporal scan statistics. In order to pre-process tasks and descriptive analysis, Microsoft Excel version 2016 (https://www.microsoft.com/) was used.

\section{Results}

\section{Descriptive analysis}

The 224,355 calls received during the study period that fitted the inclusion criteria. Mean age of patients were $43.6(\mathrm{SD}=22)$. The characteristics of the callers are listed in Table 1.

Figure 2 shows time intervals for each step of missions. The average preparation time, i.e. the time interval from patients' call to ambulance departure from the station, was almost equal for urban and rural missions (2.2 vs $2.3 \mathrm{~min}$ ). The average response time, i.e. the time it takes to reach the scene after an EMS call, was $12.2 \mathrm{~min}$ for rural missions, which is $10.1 \mathrm{~min}$ loner than urban missions with. The average time at the scene for both urban and rural missions were exactly equal (11.2 $\mathrm{min})$. Understandably, the transport time from rural locations was considerably longer (20.3 min) compared to $11.2 \mathrm{~min}$ in the urban area.

Figure 3 demonstrates the temporal variations in EMS demand during the study period, with a higher value in July and August and relatively lower one in February. Although Saturday is the first day of the week and Friday considered as weekend in Iran, this did not impact on the EMS demand that was were relatively high on
Saturdays, Wednesdays and Thursdays with two peaks: at 12 p.m. and 9 p.m.

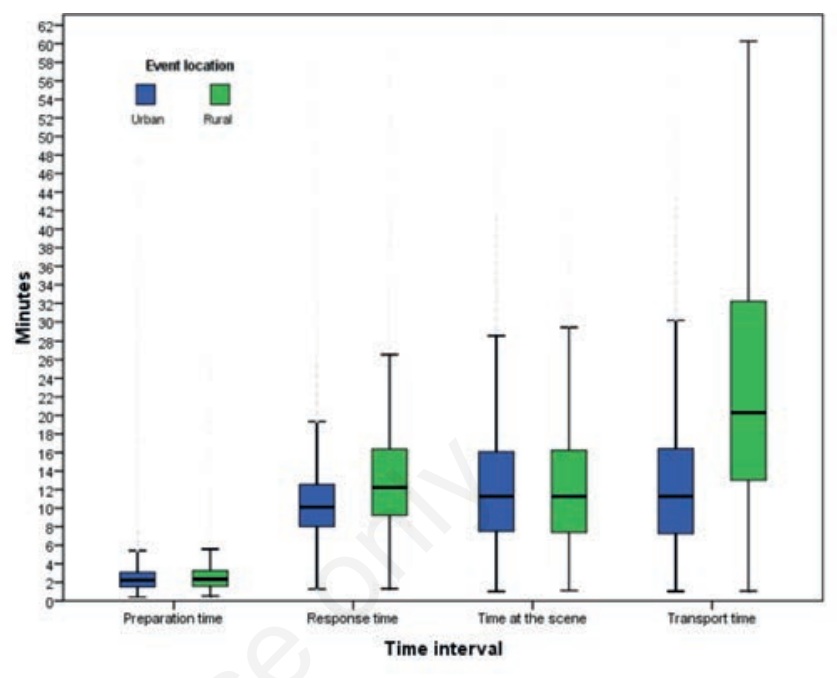

Figure 2. Box plots of time intervals of urban and rural emergency medical services missions in Mashhad, Iran from Jun 2018 to May 2019. The preparation time is the time interval from the call made to the ambulance departure from the station.

Table 1. Characteristics of emergency medical services callers in Mashhad, Iran from Jun 2018 to May 2019.

\begin{tabular}{lcc} 
Characteristics & Number of patients & $\%$ \\
Location & 224,355 & 100.0 \\
Urban & 203,072 & 90.5 \\
\hline Rural & 21,283 & 9.5 \\
Sex & 206,253 & 100.0 \\
\hline Male & 106,776 & 51.8 \\
Female & 99,477 & 48.2 \\
\hline Chief complaint & 188,885 & 100.0 \\
Altered consciousness* & 64,835 & 34.3 \\
\hline Traumatic injury & 60,822 & 32.6 \\
Chest pain and cardiac conditions & 26,016 & 14.0 \\
\hline Airway obstruction, respiratory distress & 9175 & 5.0 \\
Poisoning, intoxication, drug overdose & 7406 & 4.8 \\
\hline Abdominal pain/problems & 7291 & 3.9 \\
High/low blood pressure & 5880 & 3.8 \\
\hline Behavioural/psychiatric disorder & 3036 & 1.6 \\
Obstetric and gynaecologic emergencies & 1662 & 0.9 \\
\hline Stroke/cerebrovascular accident & 979 & 0.5 \\
Other & 1783 & 0.9 \\
\hline Mission status & 221,757 & 100.0 \\
Transfer to healthcare & 136,989 & 61.8 \\
\hline Lack of patient cooperation & 77,735 & 35.1 \\
Treated and released & 4258 & 1.9 \\
\hline Dead at scene & 2775 & 1.2 \\
\hline
\end{tabular}

*Syncope, shock, seizure and diabetic emergencies. 


\section{Thematic mapping}

Figure 4 demonstrates the geographical distribution of emergency requests at the census level divided into the six time intervals of the day. The highest smoothed incidence rates of requests were concentrated in the south-eastern part of the city at all times.

\section{Spatial and temporal clustering}

\section{Purely spatial clustering}

Using SaTScan software v.9.7, seven significant $(\mathrm{P}=0.05)$ spatial clusters of EMS requests were detected (Figure 5). These hotspot areas were categorised as most likely clusters or secondary clusters based on the P-value and RR. The first cluster incorporated 52 census tracts containing a residential population of 31,912 and 8012 emergency cases located in the downtown area. The sec- ond cluster involved a larger area with 112 census tracts with a population of 230,570 and 13,416 cases. Although this most western cluster had an observed/expected ratio of 1.37 times it is far from the 7.81 recorded for the first cluster in the downtown area. The third cluster almost covered the second one, reinforcing the importance of the area in terms of EMS requests. Other secondary likely clusters were mainly distributed in the southern part of the city. Cluster summary information is provided in the text boxes (Figure 5), with detailed description available in the supplementary Table S1.

\section{Purely temporal clustering}

Table 2 shows the results of a purely temporal analysis of EMS requests during the different four-hour periods of the day. The results indicate that high-rate clusters of EMS requests were predominantly distributed from 11:00 a.m. to 10:59 p.m. This tempo-

Table 2. Detected clusters of purely temporal emergency medical services requests in Mashhad, Iran from June 2018 to May 2019.

\begin{tabular}{|c|c|c|c|c|c|c|c|c|}
\hline Type of cluster & $\begin{array}{l}\text { Time } \\
\text { coverage }\end{array}$ & $\begin{array}{c}\text { Observed } \\
\text { number of cases }\end{array}$ & $\begin{array}{l}\text { Expected } \\
\text { number of cases }\end{array}$ & $\begin{array}{l}\text { Annual number } \\
\text { of cases } / 100,000\end{array}$ & $\mathrm{OE}$ & LLR & $\mathbf{R R}$ & P-value \\
\hline Most likely & $\begin{array}{l}\text { 11:00 a.m. - 02:59 p.m. } \\
\text { 03:00 p.m. - 06:59 p.m. } \\
\text { 07:00 p.m. - 10:59 p.m. }\end{array}$ & 79,444 & $63,512.50$ & 322,272 & 1.25 & 4039.3 & 1.67 & 0.001 \\
\hline
\end{tabular}

A cluster is statistically significant when its test statistic is greater than the critical value, which is 8.49 for 0.0001 significance level according to Gumbel critical values. OE, observed/expected; LLR, Log likelihood ratio; $\mathrm{RR}$, relative risk.

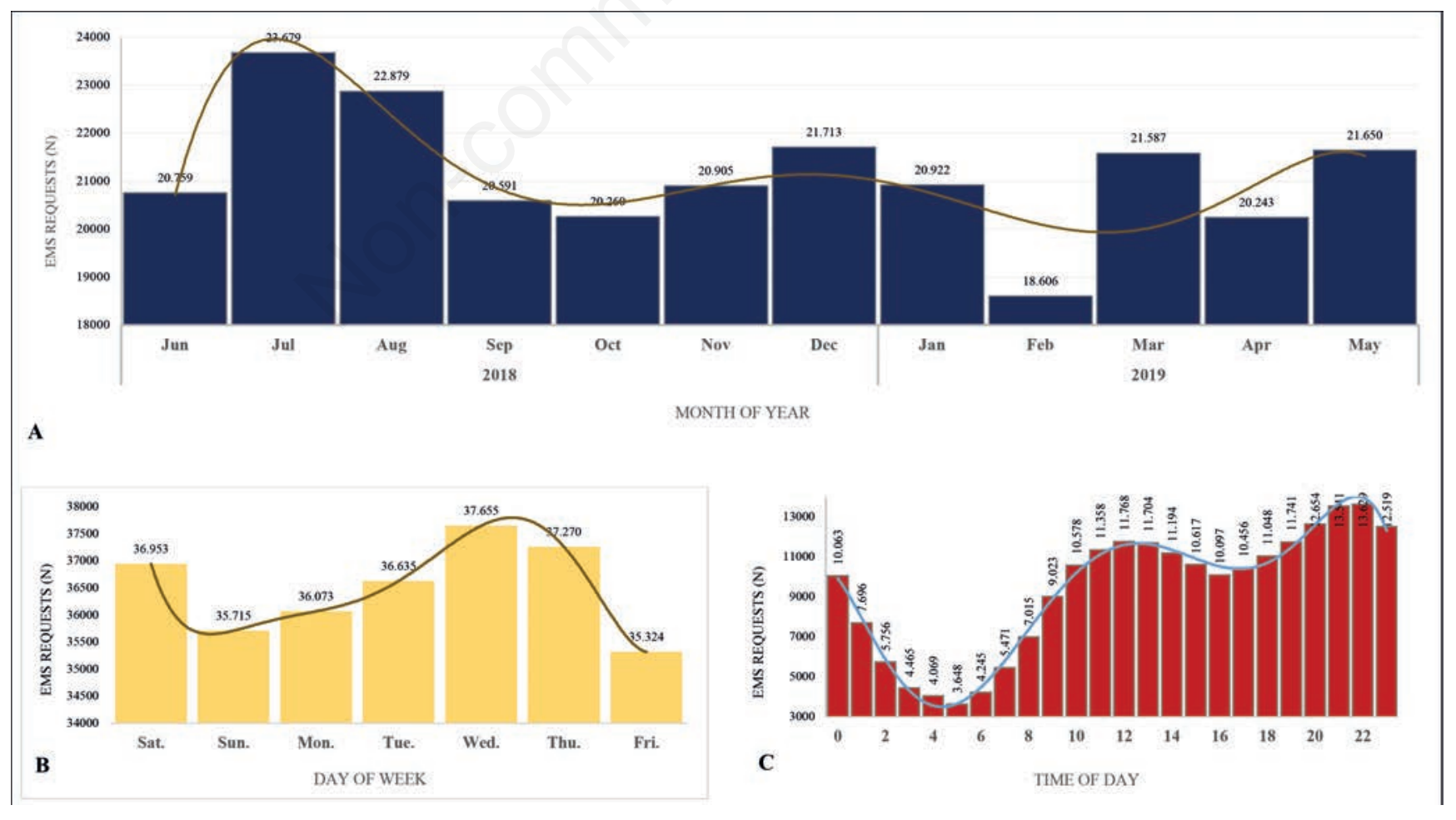

Figure 3. The temporal distribution of emergency medical services (EMS) requests in Mashhad, Iran from June 2018 to May 2019. A) Monthly variation; $B$ ) weekly variation; C) diurnal variation. 
ral cluster had an observed/expected ratio of 1.37 times with a RR of 1.67 .

\section{Space-time clustering}

Figure 6 displays clusters of EMS requests in both spatial and temporal dimensions. Two clusters were identified, both of them between 11:00 a.m. to 10:59 p.m., which is in accordance with the purely temporal clustering results. While cluster 1 included a relatively small area with 54 census tracts and 31,912 people in the eastern part of the city (downtown), cluster 2 covered a wider area with 168 census tracts and 375,591 people in the west of the city. Similar to the purely spatial scan statistics, the result of observed/expected cases for the first cluster was significantly higher than the second one (9.82 vs 1.54). Cluster summary information is provided in the text boxes (Figure 6), with detailed description available in the supplementary Table S2.

\section{Spatial variation in temporal trends}

Figure 7 demonstrates the result of SVTT for areas with higher temporal trends of EMS requests. According to this figure, two
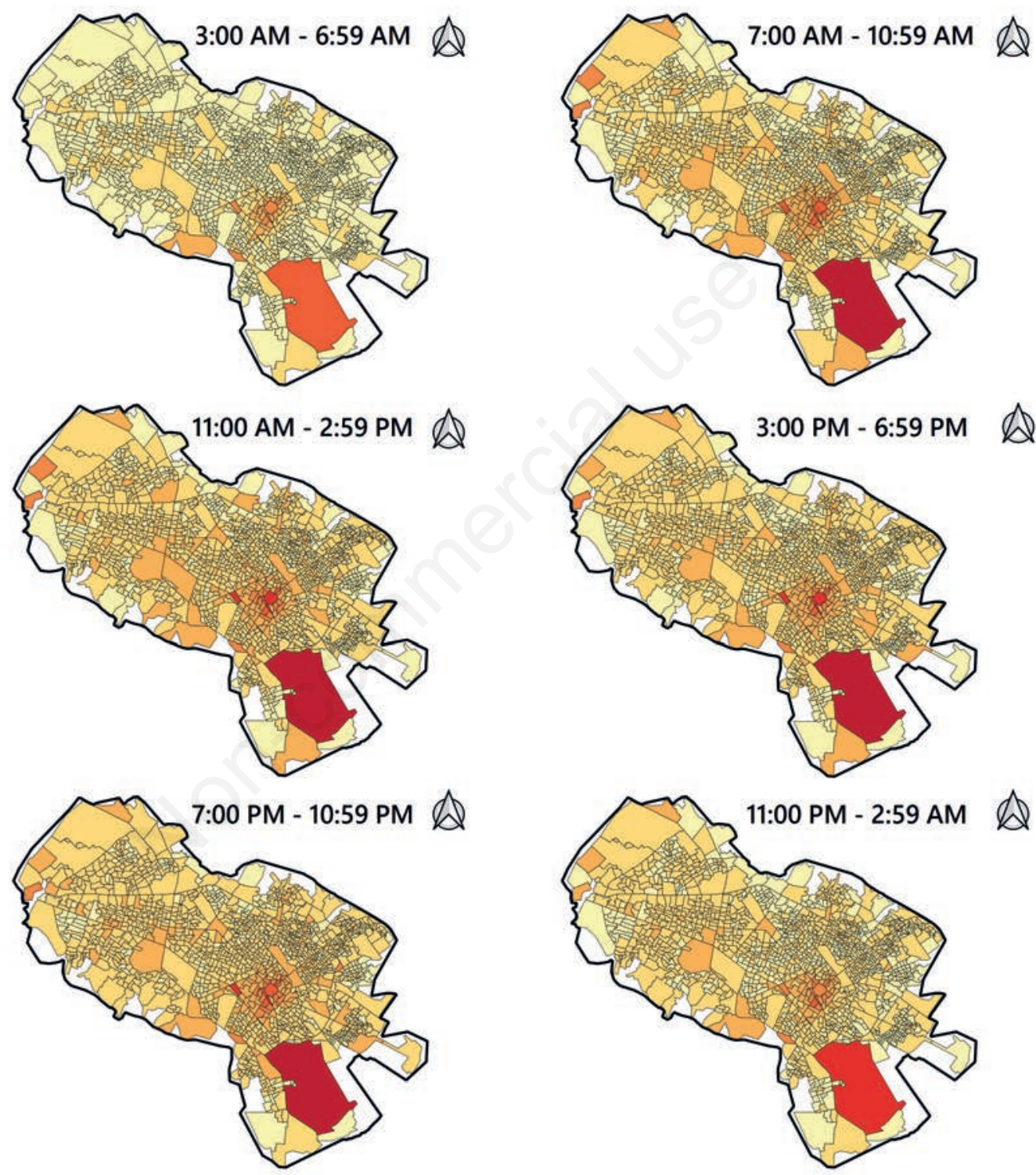

EBS rates of EMS requests per 10000
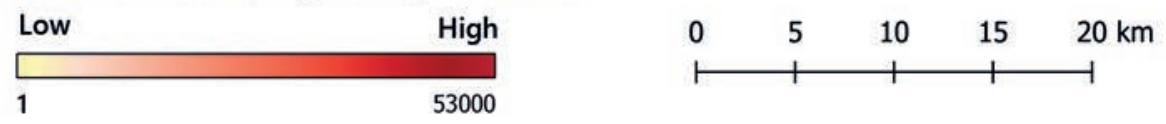

Figure 4. Empirical Bayesian Smoothed rates of emergency medical services (EMS) requests in Mashhad, Iran from June 2018 to May 2019. 
high-trend clusters in EMS requests were found to be statistically significant. The first high-trend cluster was identified in the Northeast of Mashhad with almost 655,000 residents and the second high-trend cluster in the South-east of the city with almost 69,000 populations. The inside time trends for these clusters were $15.2 \%$ and $21 \%$ while the outside time trends were $11.4 \%$ and $12.04 \%$, respectively. Cluster summary information is available in the supplementary Table S3.

\section{Convergence of emergency medical services requests and response time clusters}

Figure 8 demonstrates the convergence of purely spatial clusters of EMS requests and clusters of high response time of ambulance missions. Both were categorised as primary and secondary clusters based on P-value and RR. While they were near the centre of the city, the response time clusters were found adjacent to the border parts. Accordingly, the second cluster of high response time had no convergence with the emergency requests clusters; the clusters had also a minor overlap. Cluster summary information are provided in the text boxes (Figure 8), with detailed description available in the supplementary Table S4.

\section{Discussion}

We investigated the spatial epidemiology of EMS requests in Mashhad in 2018 and 2019 using scan statistics and descriptive epidemiological methods. The results identified a significant variation of EMS requests over space and time. To the best of our knowledge, this is the first study in the city of Mashhad to assess spatiotemporal patterns of EMS requests.

The rate of EMS requests in urban area of Mashhad was 53.4 calls/1000 citizens and year, which is very close to that in Izmir, Turkey [56.4 calls/1000 citizens and year (Sariyer et al., 2017)] and Copenhagen, Denmark [60 calls/1000 citizens and year (Moller et al., 2015)] but higher than Singapore [with 20.5 calls/1000 and year (Ong et al., 2009)].

Traumatic injuries and altered level of consciousness (includ-

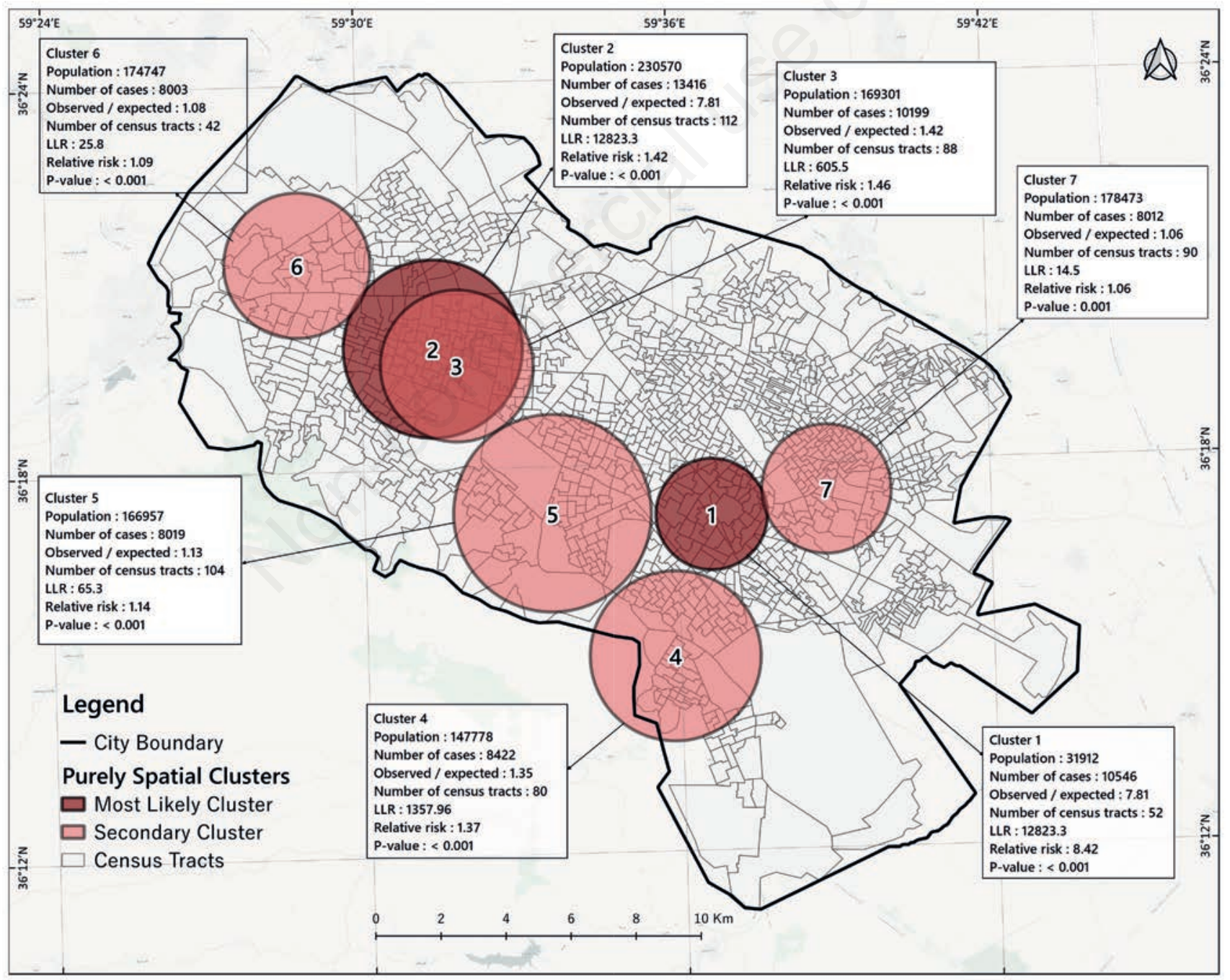

Figure 5. Purely spatial clusters of emergency medical services requests in Mashhad, Iran from June 2018 to May 2019. 
ing syncope, shock, seizure and diabetic emergencies) each accounted for almost one third of the chief complaints. This result is in accordance with a study in USA that reported that these two factors were the most frequent complaints with $23.4 \%$ and $21.7 \%$ of the total requests (Mueller et al., 2016). Interestingly, almost 35 percent of callers refused pre-hospital care. One of the common reasons for this was the wish of many patients to be transferred to a specific hospital. In Iran, ambulance staff are not allowed to transfer patients to private hospitals except for some specific reasons. Also, sometimes callers cannot wait for ambulance arrival and try to transfer patients with their personal vehicles. There is a need for more education and information to the public about the situations when they need to call ambulance services and also the associated risks of transporting a patient in a car or van lacking trained staff.

The standard response time is defined as eight min in most settings (Berlin and Liebman, 1974) which is directly related to higher survival rate and reduced mortality (Blackwell and Kaufman,
2002). This figure was higher in Mashhad by almost two min in the city area and four min in rural areas. The time spent at the scene is another important criterion in assessing the EMS performance. The global gold standard for this criterion is 10 min (Raeissi, 2012). Patients should be managed in such a way so as to minimise delays in their transfer to clinical centres. The average time spent at the scene in our study was about one min longer than the international standard.

Our study showed some temporal variation in EMS requests by month, weekday and time of day. Exact reasons for the variations are unknown but the results may generate hypotheses and have implications for the management of EMS resources. Our study identified increases in the number of emergency requests received for different temporal periods; for example, we found an increased incidence at certain times: i) around mid-day and evening/nighttime (specifically, 8:00 p.m. - 11:59 p.m.); ii) on Saturdays and Wednesdays; and iii) in the months of July and August. Other studies found similar patterns in this regard. In some studies the weekly

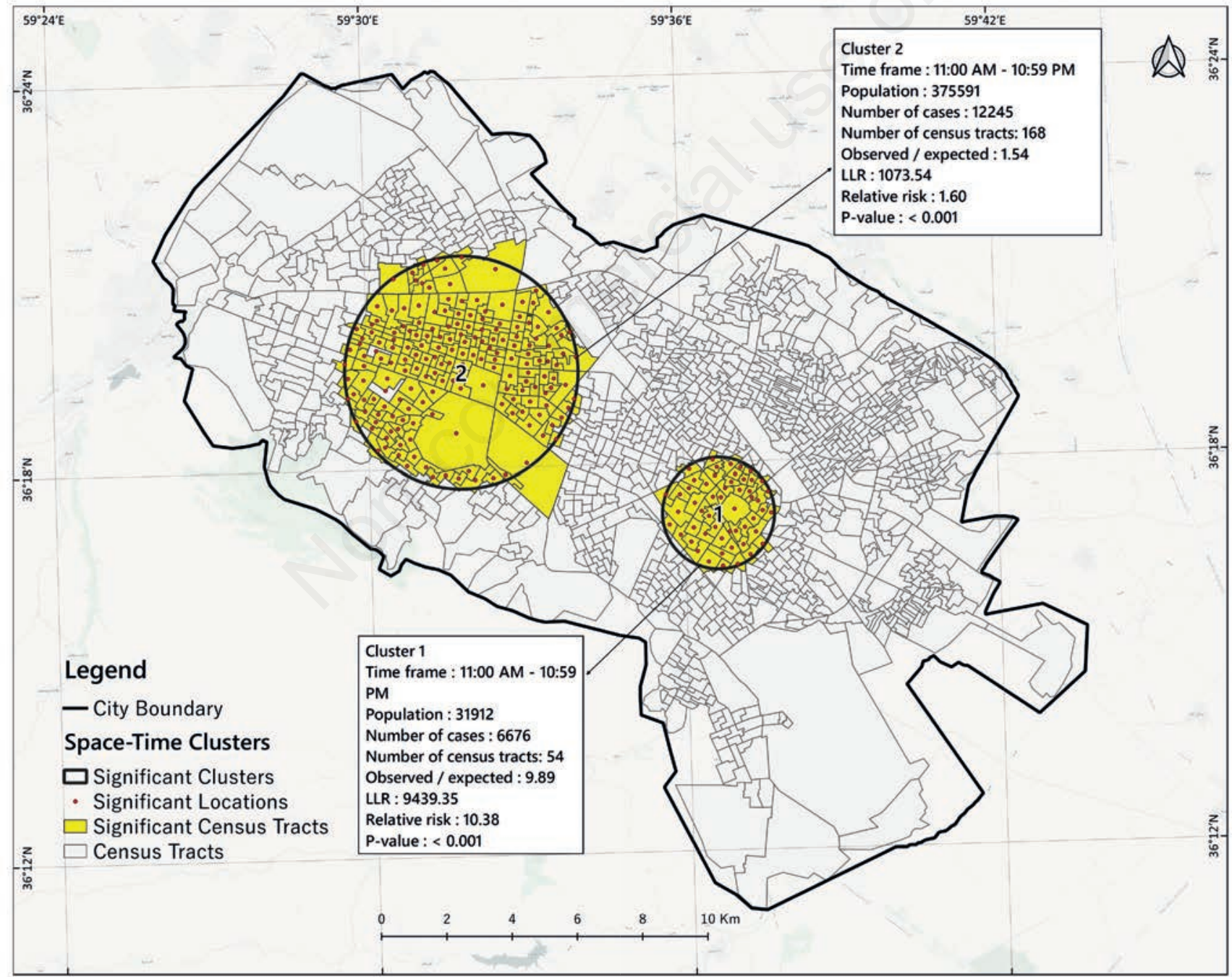

Figure 6. Detected significant spatio-temporal clusters based on space-time permutation scan statistic model for areas with high rates of emergency medical services requests rates in Mashhad, Iran from June 2018 to May 2019. Each circle specifies a significant cluster area in a specific time interval. 
pattern of peak incidence rate was identified as occurring on Mondays (Ong et al., 2009; Sariyer et al., 2017). In our study Saturdays had relatively higher EMS demand as it is the first day of week in Iran but somewhat surprisingly Wednesdays had the highest demand for pre-hospital emergency care. For diurnal and monthly variations, our findings are not much different than other studies (Willich et al., 1994; Arntz et al., 2001; Gruska et al., 2005; Ong et al., 2009).

Based on the thematic maps of EMS requests, downtown and the south-eastern part of the city had higher rates in all of the periods of the day (Figure 4). The reason for the higher request rate in downtown is because this is an area near the Holy Shrine, a focal destination for tourists and pilgrimage. Downtown Mashhad has a high population density and heavy traffic congestion in this area as compared to the rest of the city. On the other hand, EMS requests were also high in the south-eastern part of the city which includes the airport and a large census tract with a lower population density.
This could be due to the higher number of travellers to and from the airport who are not residents of this census tract.

Based on the purely spatial analysis, seven areas mostly in centre and southern part of the city identified as hotspots of emergency events (Figure 5). The figure confirms that downtown area has statistically higher demand of EMS services. The considerably higher values of observed/ expected cases and relative risk in the downtown area compared to other clusters demonstrate the unique characteristic of this location. The results of spatial scan statistics did not identify the south-eastern census tract (the airport) as a hotspot which was in red colour in the thematic map of EBS rates. This shows that having a high rate does not necessarily lead to being a spatial cluster.

Results of the purely temporal analysis identified three out of six time intervals of the day (11:00 a.m. to 10:59 p.m.) as temporal clusters of EMS demand. Although we did not find any study using temporal clustering analysis for diurnal variations, it was expected

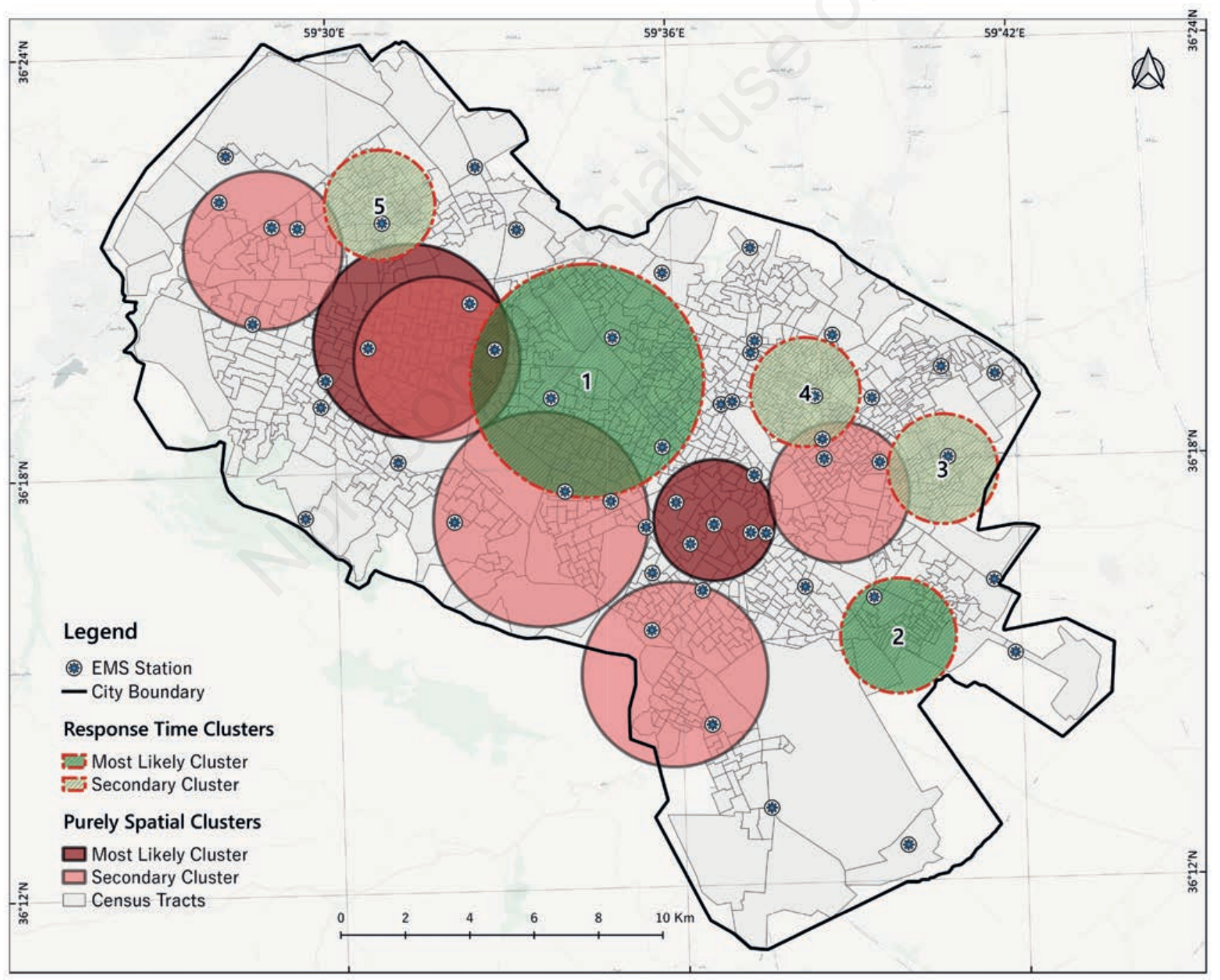

Figure 7. Detected significant clusters based on spatial variation in temporal trends of high emergency medical services (EMS) request rates in Mashhad, Iran, Jun 2018 to May 2019. Each circle specifies a significant clustered area in a specific time interval. 
that temporal clusters would be during certain hours of the day. The lower number of EMS requests at night and on Fridays (weekend in Iran) showed the association of daily activities with emergency events. The EMS system should be at its maximum capacity during these times to serve potential patients.

Based on the spatio-temporal clusters presented in this study (Figure 6), two clusters were detected to both occur between 11:00 a.m. to $10: 59$ p.m. This analysis is the combination of two prior analyses as it considers both spatial and temporal dimensions. The two identified clusters were similar to the most likely clusters in purely spatial clustering and the time intervals are equal to the detected time intervals in purely temporal clustering. Again, the considerably higher statistics for the downtown area represents the higher demand around the Holy Shrine, which is much more crowded in daytime.

The two areas found by the SVTT technique are with higher variations during the time intervals of the day (Figure 7). Higher variations of emergency requests in these areas need different approaches to supplying the right set of service policies by the associated authorities. For example, dynamic allocation of ambulances here might cover the variation of demand for emergency services.

We can infer from Figure 8 that a high number of calls in an area does not necessarily increase the ambulance response time. Although EMS demand in the downtown area of the city was a hotspot in both spatially and spatio-temporally, the average response time of ambulances was not significantly high in this area. This fact can imply that higher response time of EMS missions is associated with several factors (e.g., accessibility and road traffic) and not only due to higher number of requests and busy schedule of ambulances. Future studies can identify factors that increase the average response time of EMS missions in some areas.

The limitations experienced have mainly to do with the quality of EMS mission data, which varies as it is generated by ambulance staff in real-time, emergency and stressful situations. Secondly, the frequency of travellers/tourists in census tracts and across the study period was unknown, which will change the population at risk at some degree. There are no good data on how tourist rates

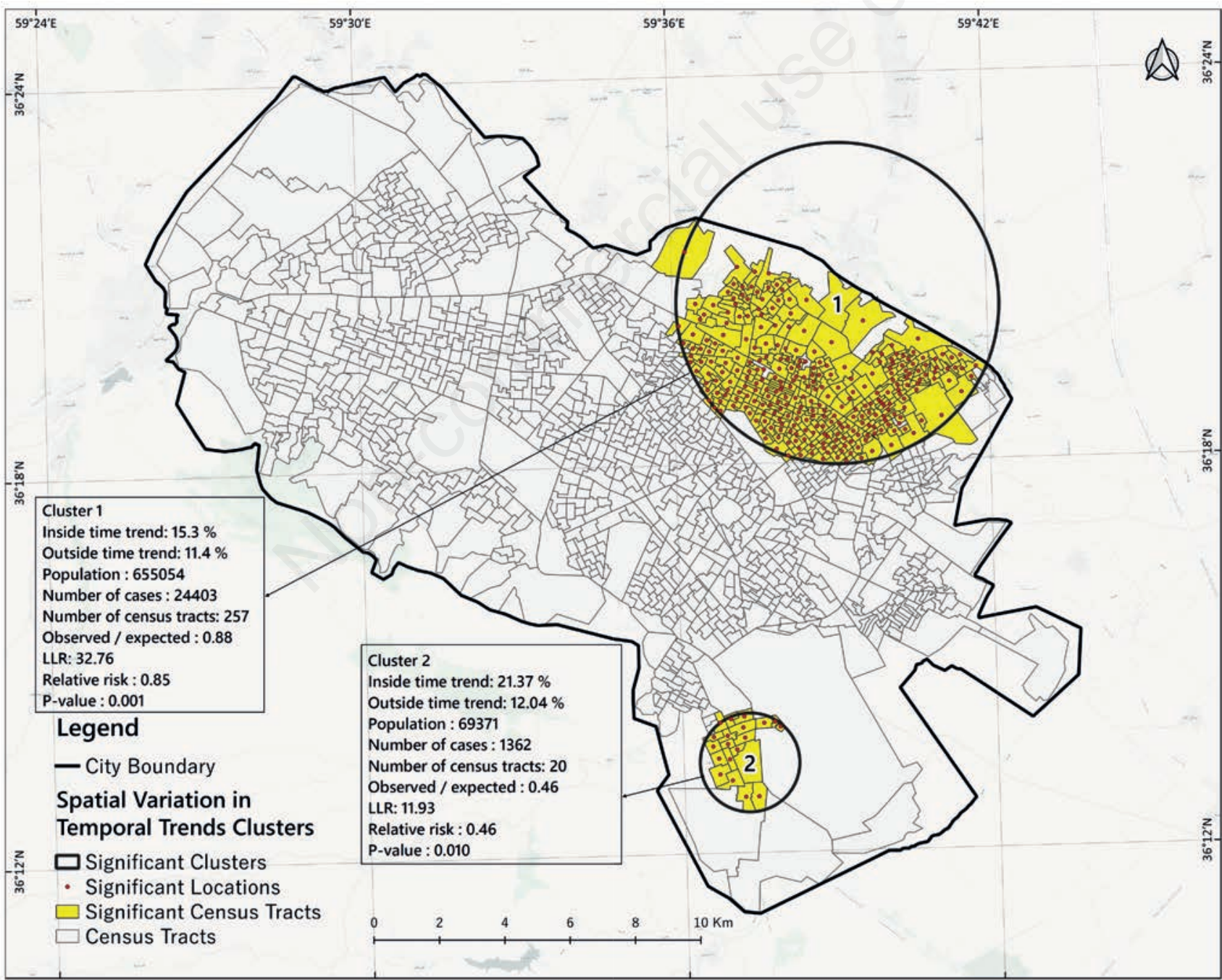

Figure 8. Comparison of detected significant spatial clusters pattern with response time clusters of emergency medical services requests in Mashhad, Iran from June 2018 to May 2019. 
within Mashhad vary by month or season, and how the religious festivals that attract visitors to the city are distributed across the year. Thirdly, this study was conducted before the COVID-19 pandemic, which had a big on the EMS requests and needs to be further explored. However, as the pandemic subsides, the situation should return what it was when the study was carried out. Finally, the issue of modifiable areal unit problem (Openshaw and Taylor, 1981) remains inherent to the studies that focus on aggregated spatial data for a single predefined area.

\section{Policy implications}

Based on our findings, we have the following suggestions. The policymakers should investigate associated factors with high EMS requests in areas detected by spatial clustering analysis. Almost one-third of EMS dispatches were terminated due to lack of patient cooperation, a fact that needs a careful review. Rates can be reduced by actions such as reforming the laws or further training of the staff. Also, some interventions should be implemented, especially during the day-time in specified areas identified by spatiotemporal analysis. These interventions can include more restricted traffic control, public awareness and improved screening of potential applicants. In addition, there are some areas that have a higher response time despite low demand. If policy makers prioritise these cluster areas in allocating ambulance resources, lower mortality and morbidity could be achieved.

\section{Conclusions}

EMS requests in Mashhad demonstrate clear spatial and temporal gradient. Although the downtown area has seen significantly higher volume of EMS enquiries, especially in the daytime, the average EMS response times are relatively acceptable. On the other hand, some areas with a normal rate of ambulance calls have significantly longer response time. The use of GIS and spatial analyses in modelling and quantifying EMS requests enables policy makers to design tailored interventions to improve quality of service. This study can serve as a model for analysis of other public health services provided in urban areas.

\section{References}

Adin A, Lee D, Goicoa T, Ugarte M D, 2019. A two-stage approach to estimate spatial and spatio-temporal disease risks in the presence of local discontinuities and clusters. Stat Methods Med Res 28:2595-613.

Ahmadian L, Salehi F, Bahaadinbeigy K, 2020. Application of geographic information systems in maternal health: a scoping review. East Mediterr Health J 26:1403-14.

Aringhieri R, Bruni ME, Khodaparasti S, Van Essen JT, 2017. Emergency medical services and beyond: Addressing new challenges through a wide literature review. Comput Oper Res 78:349-68.

Arntz H-R, Müller-Nordhorn J, Willich SN, 2001. Cold Monday mornings prove dangerous: epidemiology of sudden cardiac death. Curr Opin Crit Care 7(3).

Azimi A, Bagheri N, Mostafavi SM, Furst MA, Hashtarkhani S, Amin FH, Eslami S, Kiani F, Vafaeinezhad R, Akbari T, Golabpour A, Kiani B, 2021. Spatial-time analysis of cardiovascular emergency medical requests: enlightening policy and practice. BMC Public Health 21:7.
Bahadori M, Nasiripur A, Tofighi S, Gohari M, 2010. Emergency medical services in Iran: An overview. Australas Med J 3:335-9

Bahrami MA, Maleki A, Ranjbar Ezzatabadi M, Askari R, Ahmadi Tehrani GH, 2011. Pre-hospital emergency medical services in developing countries: A case study about EMS response time in Yazd, Iran. Iran Red Crescent Med J 13:735-8.

Bassil KL, Cole DC, Moineddin R, Craig AM, Lou WY, Schwartz B, Rea E, 2009. Temporal and spatial variation of heat-related illness using 911 medical dispatch data. Environ Res 109:600-6.

Berlin GN, Liebman JC, 1974. Mathematical analysis of emergency ambulance location. Socio-Econ Plan Sci 8:323-8.

Bidari A, Abbasi S, Farsi D, Saeidi H, Mofidi M, Radmehr M, Rezaei M , Ashayeri N, 2007. Quality assessment of prehospital care service in patients transported to Hazrat-E-Rasoul Akram Hospital. Med J Tabriz Univ Med Sci 29:43-6.

Bigdeli M, Khorasani-Zavareh D, Mohammadi R, 2010. Pre-hospital care time intervals among victims of road traffic injuries in Iran. A cross-sectional study. BMC Public Health 10:406.

Blackwell TH, Kaufman JS, 2002. Response time effectiveness: comparison of response time and survival in an urban emergency medical services system. Acad Emerg Med 9:288-95.

De Carvalho AG, Guimaraes Luz JG, Leite Dias JV, Tiwari A, Steinmann P, Ignotti E, 2020. Hyperendemicity, heterogeneity and spatial overlap of leprosy and cutaneous leishmaniasis in the southern Amazon region of Brazil. Geospat Health 15:892.

Gruska M, Gaul GB, Winkler M, Levnaic S, Reiter C, Voracek M, Kaff A, 2005. Increased occurrence of out-of-hospital cardiac arrest on Mondays in a community-based study. Chronobiol Int 22:107-20.

Haddadi M, Sarvar M, Soori H, Ainy E, 2017. The pattern of prehospital medical service delivery in Iran; a cross sectional study. Emerg Tehran 5:e57.

Hashtarkhani S, Kiani B, Bergquist R, Bagheri N, Vafaeinejad R, Tara M, 2020. An age-integrated approach to improve measurement of potential spatial accessibility to emergency medical services for urban areas. Int J Health Plann Manage 35:788-98.

Kafashpor A, Ghasempour Ganji SF, Sadeghian S, Johnson LW, 2018. Perception of tourism development and subjective happiness of residents in Mashhad, Iran. Asia Pac J Tour Res 23:521-31.

Kulldorff M, 1997. A spatial scan statistic. Commun Stat Theory Methods 26:1481-96.

Leonardsen ACL, Helgesen AK, Ulvøy L, Grøndahl VA, 2021. Prehospital assessment and management of postpartum haemorrhage-healthcare personnel's experiences and perspectives. BMC Emerg Med 21:Article 98.

Mackenzie EJ, Rivara FP, Jurkovich GJ, Nathens AB, Frey KP, Egleston BL, Salkever DS, Scharfstein DO, 2006. A national evaluation of the effect of trauma-center care on mortality. N Engl J Med 354:366-78.

Manton KG, Woodbury MA, Stallard E, Riggan WB, Creason JP, Pellom AC, 1989. Empirical Bayes procedures for stabilizing maps of U.S. cancer mortality rates. J Am Stat Assoc 84:637-50.

Mawani M, Kadir M M, Azam I, Mehmood A, Mcnally B, Stevens K, Nuruddin R, Ishaq M, Razzak J A, 2016. Epidemiology and outcomes of out-of-hospital cardiac arrest in a developing country-a multicenter cohort study. BMC Emerg Med 16:Article 28.

Mena C, Sepulveda C, Fuentes E, Ormazabal Y, Palomo I, 2018. Spatial analysis for the epidemiological study of cardiovascular diseases: a systematic literature search. Geospat Health $13: 587$

Mohammadebrahimi S, Mohammadi A, Bergquist R, Dolatkhah F, 
Olia M, Tavakolian A, Pishgar E, Kiani B, 2021. Epidemiological characteristics and initial spatiotemporal visualisation of COVID-19 in a major city in the Middle East. BMC Public Health 21:1373.

Moller TP, Ersboll AK, Tolstrup JS, Ostergaard D, Viereck S, Overton J, Folke F, Lippert F, 2015. Why and when citizens call for emergency help: an observational study of 211,193 medical emergency calls. Scand J Trauma Resusc Emerg Med 23: Article 88.

Moraga P, Kulldorff M, 2016. Detection of spatial variations in temporal trends with a quadratic function. Stat Methods Med Res 25:1422-37.

Mueller LR, Donnelly JP, Jacobson KE, Carlson JN, Mann NC, Wang HE, 2016. National characteristics of emergency medical services in frontier and remote areas. Prehosp Emerg Care 20:191-9.

Naus JL, 1965. Clustering of random points in two dimensions. Biometrika 52:263-6.

Nichol G, Thomas E, Callaway CW, Hedges J, Powell JL, Aufderheide TP, Rea T, Lowe R, Brown T, Dreyer J, Davis D, Idris A, Stiell I, 2008. Regional variation in out-of-hospital cardiac arrest incidence and outcome. J Am Med Assoc 300:1423-31.

Nykiforuk CI, Flaman LM, 2011. Geographic information systems (GIS) for Health Promotion and Public Health: a review. Health Promot Pract 12:63-73.

Ong ME, Ng FS, Overton J, Yap S, Andresen D, Yong DK, Lim SH, Anantharaman V, 2009. Geographic-time distribution of ambulance calls in Singapore: utility of geographic information system in ambulance deployment (CARE 3). Ann Acad Med Singap 38:184-91.

Openshaw S, Taylor PJ, 1981. The modifiable areal unit problem. In: Wrigley N and Bennett R (Eds.), Quantitative geography: a British view. Routledge and Kegan Paul, London, UK, pp. 60-69.

Peters J, Hall GB, 1999. Assessment of ambulance response performance using a geographic information system. Social Sci Med 49:1551-66.

Pringle DG, 1996. Mapping disease risk estimates based on small numbers: An assessment of empirical bayes techniques. Econ Social Rev 27:341-63.

Punyapornwithaya V, Sansamur C, Charoenpanyanet A, 2020. Epidemiological characteristics and determination of spatiotemporal clusters during the 2013 dengue outbreak in Chiang
Mai, Thailand. Geospat Health 15(2).

Raeissi P, 2012. The relationship between job characteristics of emergency medical technicians and scene time in traumatic injuries. IJMMS 4:186-91.

Robertson IG, 1999. Spatial and multivariate analysis, random sampling error, and analytical noise: empirical Bayesian methods at Teotihuacan, Mexico. Am Antiquity 137-52.

Sariyer G, Ataman MG, Akay S, Sofuoglu T, Sofuoglu Z, 2017. An analysis of emergency medical services demand: time of day, day of the week, and location in the city. Turk J Emerg Med 17:42-7.

Schuurman N, Cinnamon J, Crooks VA, Hameed SM, 2009. Pedestrian injury and the built environment: an environmental scan of hotspots. BMC Public Health 9:233.

Silva AEP, Chiaravalloti Neto F, Conceicao GMS, 2020. Leptospirosis and its spatial and temporal relations with natural disasters in six municipalities of Santa Catarina, Brazil, from 2000 to 2016. Geospat Health 15:903.

Spaite DW, Maio R, Garrison HG, Desmond JS, Gregor MA, Stiell IG, Cayten CG, Chew JL Jr., Mackenzie EJ, Miller DR, O'malley PJ, 2001. Emergency medical services outcomes project (EMSOP) II: developing the foundation and conceptual models for out-of-hospital outcomes research. Ann Emerg Med 37:657-63.

Tabari P, Shabanikiya H, Bagheri N, Bergquist R, Hashtarkhani S, Kiani F, Mohammadi A, Kiani B, 2020. Paediatric, pedestrian road traffic injuries in the city of Mashhad in north-eastern Iran 2015-2019: a data note. BMC Res Notes 13:363.

Wang HE, Mann NC, Jacobson KE, Ms MD, Mears G, Smyrski K, Yealy DM, 2012. National characteristics of emergency medical services responses in the United States. Prehosp Emerg Care 17:8-14.

Warden CR, Daya M, Legrady LA, 2007. Using geographic information systems to evaluate cardiac arrest survival. Prehosp Emerg Care 11:19-24.

Willich SN, Löwel H, Lewis M, Hörmann A, Arntz HR, Keil U, 1994. Weekly variation of acute myocardial infarction. Increased Monday risk in the working population. Circulation 90:87-93

Xia T, Song X, Zhang H, Song X, Kanasugi H, Shibasaki R, 2019. Measuring spatio-temporal accessibility to emergency medical services through big GPS data. Health Place 56:53-62. 